\title{
The supernova remnant W49B as seen with H.E.S.S. and Fermi-LAT ${ }^{\star}$
}

H.E.S.S. Collaboration: H. Abdalla ${ }^{1}$, A. Abramowski ${ }^{2}$, F. Aharonian ${ }^{3,4,5}$, F. Ait Benkhali ${ }^{3}$, A. G. Akhperjanian ${ }^{5,6, \dagger}$, T. Andersson ${ }^{10}$, E. O. Angüner ${ }^{7}$, M. Arrieta ${ }^{15}$, P. Aubert ${ }^{24}$, M. Backes ${ }^{8}$, A. Balzer ${ }^{9}$, M. Barnard ${ }^{1}$, Y. Becherini ${ }^{10}$, J. Becker Tjus ${ }^{11}$, D. Berge ${ }^{12}$, S. Bernhard $^{13}$, K. Bernlöhr ${ }^{3}$, R. Blackwell ${ }^{14}$, M. Böttcher ${ }^{1}$, C. Boisson ${ }^{15}$, J. Bolmont ${ }^{16}$, P. Bordas ${ }^{3}$, J. Bregeon ${ }^{17}$, F. Brun ${ }^{26, \star \star}$, P. Brun ${ }^{18}$, M. Bryan $^{9}$, T. Bulik ${ }^{19}$, M. Capasso ${ }^{29}$, J. Carr ${ }^{20}$, S. Casanova ${ }^{21,3}$, M. Cerruti ${ }^{16}$, N. Chakraborty ${ }^{3}$, R. Chalme-Calvet ${ }^{16}$, R.C. G. Chaves ${ }^{17,22}$, A. Chen $^{23}$, J. Chevalier $^{24}$, M. Chrétien ${ }^{16}$, S. Colafrancesco ${ }^{23}$, G. Cologna ${ }^{25}$, B. Condon ${ }^{26}$, J. Conrad $^{27}{ }^{28}$, Y. Cui ${ }^{29}$, I. D. Davids ${ }^{1,8}$, J. Decock $^{18}$, B. Degrange ${ }^{30}$, C. Deil ${ }^{3}$, J. Devin ${ }^{17}$, P. deWilt ${ }^{14}$, L. Dirson ${ }^{2}$, A. Djannati-Atai ${ }^{31}$, W. Domainko ${ }^{3}$, A. Donath ${ }^{3}$, L. O'C. Drury ${ }^{4}$, G. Dubus ${ }^{32}$, K. Dutson ${ }^{33}$, J. Dyks ${ }^{34}$, T. Edwards ${ }^{3}$, K. Egberts ${ }^{35}$, P. Eger ${ }^{3}$, J.-P. Ernenwein ${ }^{20}$, S. Eschbach ${ }^{36}$, C. Farnier $^{27,10}$, S. Fegan ${ }^{30}$, M. V. Fernandes ${ }^{2}$, A. Fiasson ${ }^{24}$, G. Fontaine ${ }^{30}$, A. Förster ${ }^{3}$, S. Funk ${ }^{36}$, M. Füßling ${ }^{37}$, S. Gabici ${ }^{31}$, M. Gajdus ${ }^{7}$, Y. A. Gallant ${ }^{17}$, T. Garrigoux ${ }^{1}$, G. Giavitto ${ }^{37}$, B. Giebels ${ }^{30}$, J. F. Glicenstein ${ }^{18}$, D. Gottschall ${ }^{29}$, A. Goyal ${ }^{38}$, M.-H. Grondin ${ }^{26}$, D. Hadasch ${ }^{13}$, J. Hahn ${ }^{3}$, M. Haupt ${ }^{37}$, J. Hawkes ${ }^{14}$, G. Heinzelmann ${ }^{2}$, G. Henri' ${ }^{32}$, G. Hermann $^{3}$,

O. Hervet ${ }^{15,46}$, J. A. Hinton ${ }^{3}$, W. Hofmann ${ }^{3}$, C. Hoischen ${ }^{35}$, M. Holler ${ }^{30}$, D. Horns ${ }^{2}$, A. Ivascenko ${ }^{1}$, A. Jacholkowska ${ }^{16}$, M. Jamrozy ${ }^{38}$, M. Janiak ${ }^{34}$, D. Jankowsky ${ }^{36}$, F. Jankowsky ${ }^{25}$, M. Jingo ${ }^{23}$, T. Jogler ${ }^{36,43, \star \star}$, L. Jouvin ${ }^{31}$, I. Jung-Richardt ${ }^{36}$, M. A. Kastendieck ${ }^{2}$,

K. Katarzyński ${ }^{39}$, U. Katz ${ }^{36}$, D. Kerszberg ${ }^{16}$, B. Khélifi ${ }^{31}$, M. Kieffer ${ }^{16}$, J. King ${ }^{3}$, S. Klepser ${ }^{37}$, D. Klochkov ${ }^{29}$, W. Kluźniak ${ }^{34}$, D. Kolitzus ${ }^{13}$, Nu. Komin ${ }^{23}$, K. Kosack ${ }^{18}$, S. Krakau ${ }^{11}$, M. Kraus ${ }^{36}$, F. Krayzel ${ }^{24}$, P. P. Krüger ${ }^{1}$, H. Laffon ${ }^{26}$, G. Lamanna ${ }^{24}$, J. Lau ${ }^{14}$, J.-P. Lees ${ }^{24}$, J. Lefaucheur ${ }^{15}$, V. Lefranc ${ }^{18}$, A. Lemière ${ }^{31}$, M. Lemoine-Goumard ${ }^{26, \star \star}$, J.-P. Lenain ${ }^{16}$, E. Leser ${ }^{35}$, T. Lohse ${ }^{7}$, M. Lorentz ${ }^{18}$, R. Liu ${ }^{3}$, R. López-Coto ${ }^{3}$ I. Lypova ${ }^{37}$, V. Marandon ${ }^{3, \star \star}$, A. Marcowith ${ }^{17}$, C. Mariaud ${ }^{30}$, R. Marx $^{3}$, G. Maurin ${ }^{24}$, N. Maxted ${ }^{14}$, M. Mayer ${ }^{7}$, P. J. Meintjes ${ }^{40}$, M. Meyer ${ }^{27}$, A. M. W. Mitchell ${ }^{3}$, R. Moderski ${ }^{34}$, M. Mohamed ${ }^{25}$, L. Mohrmann ${ }^{36}$, K. Morå $^{27}$, E. Moulin ${ }^{18}$, T. Murach ${ }^{7}$, M. de Naurois ${ }^{30}$, F. Niederwanger ${ }^{13}$, J. Niemiec ${ }^{21}$, L. Oakes ${ }^{7}$, P. O’Brien ${ }^{33}$, H. Odaka ${ }^{3}$, S. Öttl ${ }^{13}$, S. Ohm ${ }^{37}$, M. Ostrowski ${ }^{38}$, I. Oya ${ }^{37}$, M. Padovani ${ }^{17}$, M. Panter $^{3}$,

R. D. Parsons ${ }^{3}$, N. W. Pekeur ${ }^{1}$, G. Pelletier ${ }^{32}$, C. Perennes ${ }^{16}$, P.-O. Petrucci ${ }^{32}$, B. Peyaud ${ }^{18}$, Q. Piel ${ }^{24}$, S. Pita $^{31}$, H. Poon $^{3}$, D. Prokhorov ${ }^{10}$,

H. Prokoph ${ }^{10}$, G. Pühlhofer ${ }^{29}$, M. Punch ${ }^{31,10}$, A. Quirrenbach ${ }^{25}$, S. Raab ${ }^{36}$, A. Reimer ${ }^{13}$, O. Reimer ${ }^{13}$, M. Renaud ${ }^{17}$, R. de los Reyes ${ }^{3}$,

F. Rieger ${ }^{3,41}$, C. Romoli ${ }^{4}$, S. Rosier-Lees ${ }^{24}$, G. Rowell ${ }^{14}$, B. Rudak ${ }^{34}$, C. B. Rulten ${ }^{15}$, V. Sahakian ${ }^{6,5}$, D. Salek ${ }^{42}$, D. A. Sanchez ${ }^{24}$,

A. Santangelo ${ }^{29}$, M. Sasaki ${ }^{29}$, R. Schlickeiser ${ }^{11}$, F. Schüssler ${ }^{18}$, A. Schulz ${ }^{37}$, U. Schwanke ${ }^{7}$, S. Schwemmer ${ }^{25}$, M. Settimo ${ }^{16}$, A. S. Seyffert ${ }^{1}$, N. Shafi ${ }^{23}$, I. Shilon ${ }^{36}$, R. Simoni ${ }^{9}$, H. Sol ${ }^{15}$, F. Spanier ${ }^{1}$, G. Spengler ${ }^{27}$, F. Spies ${ }^{2}$, Ł. Stawarz $^{38}$, R. Steenkamp ${ }^{8}$, C. Stegmann ${ }^{35,37}$, F. Stinzing ${ }^{36, \dagger}$, K. Stycz ${ }^{37}$, I. Sushch ${ }^{1}$, J.-P. Tavernet ${ }^{16}$, T. Tavernier ${ }^{31}$, A. M. Taylor ${ }^{4}$, R. Terrier ${ }^{31}$, L. Tibaldo $^{3}$, D. Tiziani ${ }^{36}$, M. Tluczykont ${ }^{2}$, C. Trichard ${ }^{20}$, R. Tuffs ${ }^{3}$, Y. Uchiyama ${ }^{44}$, D. J. van der Walt ${ }^{1}$, C. van Eldik ${ }^{36}$, C. van Rensburg ${ }^{1}$, B. van Soelen ${ }^{40}$, G. Vasileiadis ${ }^{17}$, J. Veh $^{36}$, C. Venter ${ }^{1}$, A. Viana $^{3}$, P. Vincent ${ }^{16}$, J. Vink ${ }^{9}$, F. Voisin ${ }^{14}$, H. J. Völk ${ }^{3}$, T. Vuillaume ${ }^{24}$, Z. Wadiasingh ${ }^{1}$, S. J. Wagner ${ }^{25}$, P. Wagner ${ }^{7}$, R.M. Wagner ${ }^{27}$, R. White ${ }^{3}$, A. Wierzcholska ${ }^{21}$, P. Willmann ${ }^{36}$, A. Wörnlein ${ }^{36}$, D. Wouters ${ }^{18}$, R. Yang ${ }^{3}$, V. Zabalza ${ }^{33}$, D. Zaborov ${ }^{30}$, M. Zacharias $^{25}$, A. A. Zdziarski $^{34}$, A. Zech ${ }^{15}$, F. Zefi ${ }^{30}$, A. Ziegler ${ }^{36}$, and N. Żywucka ${ }^{38}$

From Fermi-LAT Collaboration: J. Katsuta ${ }^{45, \star \star}$

(Affiliations can be found after the references)

Received 26 November 2015 / Accepted 24 August 2016

\section{ABSTRACT}

The supernova remnant (SNR) W49B originated from a core-collapse supernova that occurred between one and four thousand years ago, and subsequently evolved into a mixed-morphology remnant, which is interacting with molecular clouds (MC). Gamma-ray observations of SNR$\mathrm{MC}$ associations are a powerful tool to constrain the origin of Galactic cosmic rays, as they can probe the acceleration of hadrons through their interaction with the surrounding medium and subsequent emission of non-thermal photons. We report the detection of a $\gamma$-ray source coincident with W49B at very high energies (VHE; $E>100 \mathrm{GeV}$ ) with the H.E.S.S. Cherenkov telescopes together with a study of the source with five years of Fermi-LAT high-energy $\gamma$-ray $(0.06-300 \mathrm{GeV})$ data. The smoothly connected, combined source spectrum, measured from $60 \mathrm{MeV}$ to multi-TeV energies, shows two significant spectral breaks at $304 \pm 20 \mathrm{MeV}$ and $8.4_{-25}^{+2.2} \mathrm{GeV}$; the latter is constrained by the joint fit from the two instruments. The detected spectral features are similar to those observed in several other SNR-MC associations and are found to be indicative of $\gamma$-ray emission produced through neutral-pion decay.

Key words. gamma rays: general - ISM: supernova remnants - ISM: clouds

\section{Introduction}

The strong shocks associated with supernova remnants (SNRs) are very good candidates for accelerating hadronic Galactic

\footnotetext{
* The H.E.S.S. gamma-ray excess map (see Fig. 1, in FITS format) of the W49 region is only available at the CDS via anonymous ftp to cdsarc.u-strasbg. fr (130.79.128.5) or via

http://cdsarc.u-strasbg.fr/viz-bin/qcat?J/A+A/612/A5

$\dagger$ Deceased.

$\star \star$ Corresponding authors: H.E.S.S. Collaboration,

e-mail: contact.hess@hess-experiment.eu
}

cosmic rays to at least $10^{15} \mathrm{eV}$ through the diffusive shock acceleration mechanism (e.g. Drury 1983). The detection of very high energy (VHE) $\gamma$-rays above $20 \mathrm{TeV}$ in objects such as RX J1713.7-3946 (Aharonian et al. 2006b) or RCW 86 (Aharonian et al. 2009) shows that these objects can accelerate particles up to at least $100 \mathrm{TeV}$. However, whether the bulk of accelerated particles that radiate in the high-energy (HE; $0.1<E<100 \mathrm{GeV})$ and very high-energy $(E>100 \mathrm{GeV}) \gamma$-ray range is of leptonic or hadronic nature is still under debate (Blasi 2013).

A growing number of SNRs interacting with molecular clouds (SNR-MC) are being revealed in the $\mathrm{GeV}$ and 
TeV $\gamma$-ray domain. This includes W44 (Abdo et al. 2010b), W28 (Aharonian et al. 2008b; Abdo et al. 2010a), CTB 37A (Aharonian et al. 2008a; Castro \& Slane 2010), and IC 443 (Albert et al. 2007; Acciari et al. 2009; Abdo et al. 2010c). Even though isolated SNRs are obviously cleaner laboratories to study cosmic-ray acceleration processes, SNR-MC associations are good candidates to test the presence of hadronic acceleration in SNRs; this is in part because the HE and VHE $\gamma$-ray emissions from the decay of $\pi^{0}$ mesons are expected to be strongly enhanced. The neutral pions, which are produced when highenergy protons (or nuclei) collide with interstellar material, each decay into two $\gamma$-rays with equal energies in the rest frame of the pion. This translates into a steep rise below $\sim 200 \mathrm{MeV}$ in the $\gamma$-ray spectral energy distribution (often referred to as the pion-decay bump). This characteristic spectral feature has been recently detected at high energies for three interacting SNRs: IC 443, W44 (Ackermann et al. 2013), and W51C (Jogler \& Funk 2016). However, whether this feature is the signature of the acceleration of freshly injected protons may be questioned, as re-acceleration of diffuse cosmic rays for a limited time period is also possible (Uchiyama et al. 2010; Cardillo et al. 2016).

In this context, the $\mathrm{W} 49$ region, discovered in the $22 \mathrm{~cm}$ survey of Westerhout (1958), represents one of the most interesting regions in the Galaxy to study cosmic-ray acceleration. This region contains two remarkable objects: a young SNR (W49B) and a star-forming region (W49A). The SNR W49B (G43.3-0.2) is another example of a SNR-MC association. It is a mixedmorphology (Rho \& Petre 1998) SNR with a bright shell of diameter $\sim 4^{\prime}$ that is resolved at radio wavelengths and centrally filled with thermal X-rays (Hwang et al. 2000). With a flux density of $38 \mathrm{Jy}$ at $1 \mathrm{GHz}$, this source is one of the brightest SNRs of the Galaxy at radio wavelengths. Extensive infrared and X-ray studies revealed that the progenitor of W49B was a supermassive star that created a wind-blown bubble in a dense molecular cloud in which the explosion occurred (Keohane et al. 2007). It has recently been shown that the progenitor of W49B experienced a jet-driven, core-collapse explosion (Lopez et al. 2013; González-Casanova et al. 2014). Observations of mid-infrared lines from shocked molecular hydrogen show that W49B is interacting with molecular clouds. Near-infrared Fe II observations revealed filamentary structures, which is evidence of radiative shocks (Keohane et al. 2007). This interaction is also suggested by H I line observations (Brogan \& Troland 2001). The age of the remnant is estimated to be between 1000 and 4000 years (Moffett \& Reynolds 1994; Hwang et al. 2000; Zhou et al. 2011) and the distance of this object is still not very well constrained. From H I absorption analyses, Radhakrishnan et al. (1972) derived a distance, which Moffett \& Reynolds (1994) later recalculated to be $\sim 8 \mathrm{kpc}$ using an updated Galactic rotation model. Brogan \& Troland (2001), using more recent VLA data, have shown that an association of W49B with the nearby star-forming region $\mathrm{W} 49 \mathrm{~A}$ is also possible, extending the range of possible distances $(8 \leq D \leq 12 \mathrm{kpc})$ for this source. More recently, Zhu et al. (2014) obtained a distance of $\sim 10 \mathrm{kpc}$.

As mentioned above, the other notable component of the W49 region is W49A (Mezger et al. 1967). This is one of the brightest giant radio H II regions in the Galaxy. This starforming region is located in the densest $\sim 15 \mathrm{pc}$ of a $10^{6} M_{\odot}$ giant molecular cloud of $\sim 100 \mathrm{pc}$ in size. It contains numerous compact and ultra-compact (UC) H II regions and its emission is equivalent to the presence of about $100 \mathrm{O} 7$ stars (Brogan \& Troland 2001). The presence of a very massive star with an initial mass of $100-180 M_{\odot}$ has been reported by
Wu et al. (2014). W49A is associated with a molecular outflow and strong $\mathrm{H}_{2} \mathrm{O}$ masers (Walker et al. 1982; Scoville et al. 1986), the proper motion of which was used by Zhang et al. (2013) to determine a distance of $11.11_{-0.69}^{+0.79} \mathrm{kpc}$. Star-forming regions are considered potential $\mathrm{VHE}^{-} \gamma$-ray emitters since they generally host massive stars that could accelerate particles to VHEs through interactive or collective wind effects. As an example, the TeV $\gamma$-ray source coincident with Westerlund 1 (Abramowski et al. 2012), detected with the High Energy Stereoscopic System (H.E.S.S.), may be the site of such processes.

In the HE domain, a bright source coincident with W49B is detected with the Fermi Large Area Telescope (LAT) (3FGL J1910.9+0906 in the Fermi-LAT 3FGL catalogue; Acero et al. 2015). This bright source was one of the 205 most significant sources after the first three months of observation ${ }^{1}$ and is one of the 360 sources detected above $50 \mathrm{GeV}$ in 80 months of data (Ackermann et al. 2016). This source is the subject of a detailed analysis presented in Abdo et al. (2010d). In that paper, the detection of $\mathrm{HE} \gamma$-ray emission was reported towards W49B at a significance of $38 \sigma$ with 17 months of data. The authors disfavoured a possible pulsar origin for the emission observed towards $\mathrm{W} 49 \mathrm{~B}$ in the $\mathrm{GeV}$ range. Furthermore, from X-ray measurements, the presence of a neutron star as the result of the collapse of the progenitor star appears unlikely (Lopez et al. 2013).

In the present paper, the detection of a source coincident with the SNR W49B in the VHE $\gamma$-ray domain with H.E.S.S. is reported. The analysis of the Fermi-LAT data is applied in this work to five years of data using an updated calibration and updated source and background models. The spectral and morphological results in the $\mathrm{GeV}$ and $\mathrm{TeV}$ regime are discussed in the context of a SNR interacting with a molecular cloud.

\section{Data analysis}

\subsection{H.E.S.S. observations and analysis}

\subsubsection{H.E.S.S. data set and analysis methods}

H.E.S.S. is an array of five imaging Cherenkov telescopes located in the Khomas Highland in Namibia $\left(23^{\circ} 16^{\prime} 18^{\prime \prime} \mathrm{S}\right.$, $16^{\circ} 30^{\prime} 01^{\prime \prime} \mathrm{E}$ ) at an altitude of $1800 \mathrm{~m}$ above sea level (Hinton 2004). The fifth telescope was added in July 2012 but observations with the upgraded system are not used in this work. With the initial four-telescope array, $\gamma$-rays are detected above an energy threshold of $\sim 100 \mathrm{GeV}$ with an angular resolution better than $0.1^{\circ}$ and an energy resolution below $15 \%$.

A standard data quality selection procedure was used to remove observations affected by bad weather or instrumental problems (Aharonian et al. 2006a). Extrapolating the FermiLAT $\gamma$-ray spectrum to higher energies, W49B is expected to have a steep spectrum in the $\mathrm{TeV}$ range; this is also a common feature for $\mathrm{GeV}$-detected SNRs interacting with molecular clouds. Therefore, observations with an energy threshold above $600 \mathrm{GeV}$, which can be determined a priori from the observation conditions, were not used in this study. This resulted in a data set comprising $75 \mathrm{~h}$ (live time) of observations towards the W49 region taken from 2004 to 2013 with a mean pointing offset of $1.1^{\circ}$ and a mean zenith angle of $37^{\circ}$. The data set comprises dedicated observations as well as observations of other nearby sources or dedicated to the survey of the Galactic plane (H.E.S.S. Collaboration 2018).

1 It was designated as 0FGL J1911.0+0905 in this so-called Bright Sources List (Abdo et al. 2009a). 


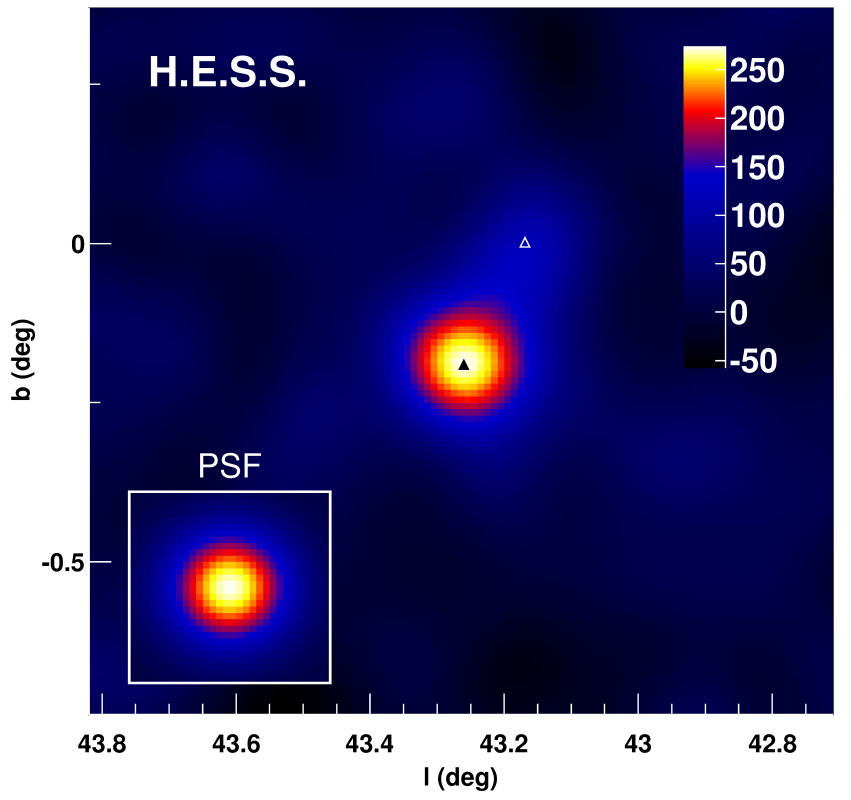

Fig. 1. $\gamma$-ray excess map of the W49 region obtained with H.E.S.S. The map is smoothed with a Gaussian of width $0.06^{\circ}$, corresponding to the $68 \%$ radius containment of the H.E.S.S. point spread function (PSF; shown in the inset). The black triangle denotes the position of the supernova remnant W49B (Green 2014). The white triangle denotes the position of W49A (Lockman 1989).

The data were analysed using the Model Analysis (de Naurois \& Rolland 2009), in which shower images of all triggered telescopes are compared to a precalculated model by means of a log likelihood optimisation. This advanced reconstruction technique improves the sensitivity with respect to more conventional techniques. The standard cuts of the Model Analysis were adopted. This set of cuts includes a minimum charge in the shower images of 60 photoelectrons resulting, for this data set, in an energy threshold of $290 \mathrm{GeV}$. This set of cuts was used for signal extraction and for the spectral and morphological analyses. The results presented in this paper have been cross-checked with the analysis methods described in Ohm et al. (2009) and Parsons \& Hinton (2014) and yield compatible results.

\subsubsection{H.E.S.S. analysis results}

The signal was extracted from a circular region with a radius of $0.1^{\circ}$ around the position of $\mathrm{W} 49 \mathrm{~B}$, taken inside the radio shell, at the position of the maximum of the emission in X-rays determined by Hwang et al. (2000), that is $\left(\ell=43.275^{\circ}, b=-0.190^{\circ}\right)$. From the correlated excess map, which is computed using the ring background subtraction method (Berge et al. 2007) and shown in Fig. 1, a significant excess of VHE $\gamma$-rays is detected at the position of W49B, with a peak significance of $12.9 \sigma$ (Eq. (17) of $\mathrm{Li} \& \mathrm{Ma} 1983)$. With the primary analysis, VHE $\gamma$-ray emission is observed towards W49A, but this observation could not be confirmed (above $5 \sigma$ ) with the cross-check analysis. Therefore, only the VHE emission coincident with W49B is discussed in the following.

In order to derive the source position at VHE and therefore the best-suited spectral extraction region, the excess map of VHE $\gamma$-rays towards the W49 region was fitted with a twosource model. This is undertaken to model the region in the best possible way, given the possible excess towards W49A, which is $\sim 0.2^{\circ}$ away from W49B (a model with only one source for the fit leads to compatible results). The model is convolved with the H.E.S.S. point spread function before being

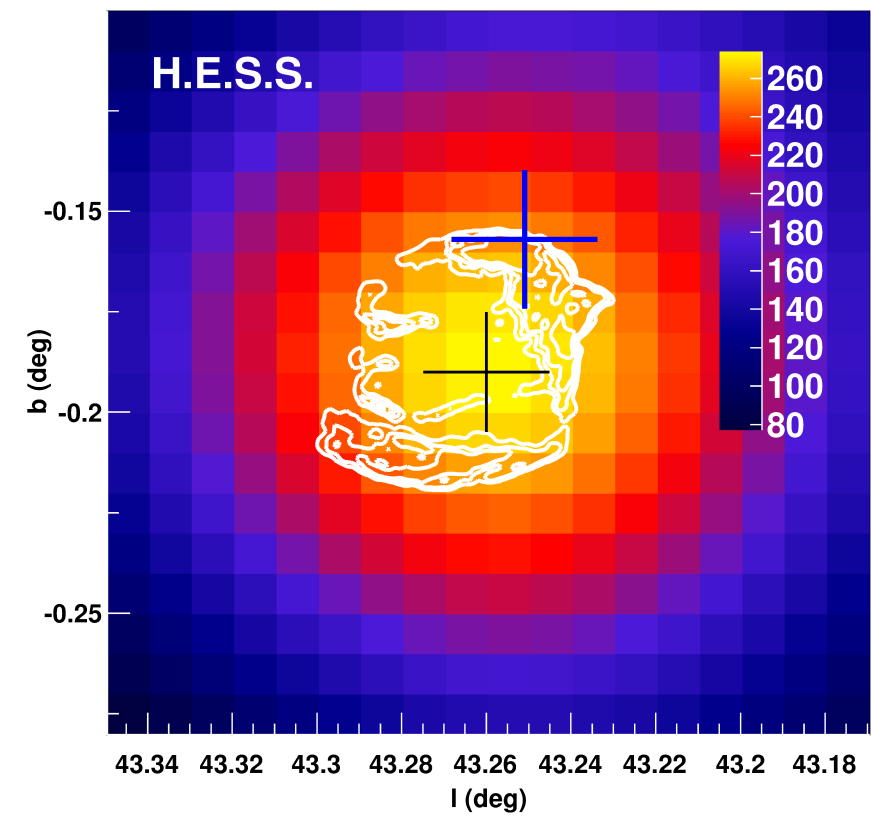

Fig. 2. Zoomed-in version of Fig. 1. The crosses show the best-fit positions of the source with H.E.S.S. (black) and Fermi-LAT (blue). The size of the crosses represents the size of the 95\% confidence level (CL) contours on the best-fit positions, including systematic uncertainties. The white contours show the radio intensity obtained at a wavelength of $20 \mathrm{~cm}$ with MAGPIS (Helfand et al. 2006).

fit to the measured excess counts. The VHE $\gamma$-ray emission towards $\mathrm{W} 49 \mathrm{~B}$ is found to be point-like with a best-fit position of $\left(\ell=43.260^{\circ} \pm 0.005_{\text {stat }}^{\circ}, b=-0.190^{\circ} \pm 0.005_{\text {stat }}^{\circ}\right)$ with a systematic error of $\pm 0.006^{\circ}$ on each axis. As can be seen in Fig. 2, the obtained position is compatible with the centre of the SNR observed in radio and X-rays (e.g. Pye et al. 1984). This position corresponds to $\alpha_{2000}=19^{\mathrm{h}} 11^{\mathrm{m}} 7.3^{\mathrm{s}}, \delta_{2000}=09^{\circ} 05^{\prime} 37.0^{\prime \prime}$ and the detected source is therefore given the identifier HESS $\mathrm{J} 1911+090$.

The reflected background subtraction method (Berge et al. 2007) was used at the best-fit position to derive the spectrum of the VHE emission coincident with W49B. From a region of radius $0.1^{\circ}$ around this position, the total number of counts is 1141 , while 16017 events are used to estimate the background with an average normalisation factor $\alpha=0.047$. This corresponds to an excess of 392.3 events detected with a significance of $13.0 \sigma$. The differential energy spectrum is derived above $290 \mathrm{GeV}$ using a forward-folding method described in Piron et al. (2001). The data are described well $\left(\chi^{2} / \mathrm{ndf}=60.6 / 61\right)$ by a power-law model $\mathrm{d} N / \mathrm{d} E=\Phi_{0}(E / 1 \mathrm{TeV})^{-\Gamma}$ with a flux normalisation $\Phi_{0}=$ $\left(3.15 \pm 0.46_{\text {stat }} \pm 0.63_{\text {sys }}\right) \times 10^{-13} \mathrm{~cm}^{-2} \mathrm{~s}^{-1} \mathrm{TeV}^{-1}$ and a spectral index of $\Gamma=3.14 \pm 0.24_{\text {stat }} \pm 0.10_{\text {sys }}$ (see Fig. 3). The integrated flux above $1 \mathrm{TeV}$ is $F(E>1 \mathrm{TeV})=\left(1.47 \pm 0.38_{\text {stat }} \pm 0.29_{\text {sys }}\right) \times$ $10^{-13} \mathrm{~cm}^{-2} \mathrm{~s}^{-1}$, corresponding to $0.65 \%$ of the Crab nebula flux above the same energy (Aharonian et al. 2006a). Two other spectral shapes were tested (log parabolic power law and power law with exponential cut-off) but they do not fit the data significantly better.

\subsection{Fermi-LAT analysis}

\subsubsection{Fermi-LAT observations and data reduction}

The LAT detects $\gamma$-ray photons by conversion into electronpositron pairs in the energy range between $20 \mathrm{MeV}$ to higher 


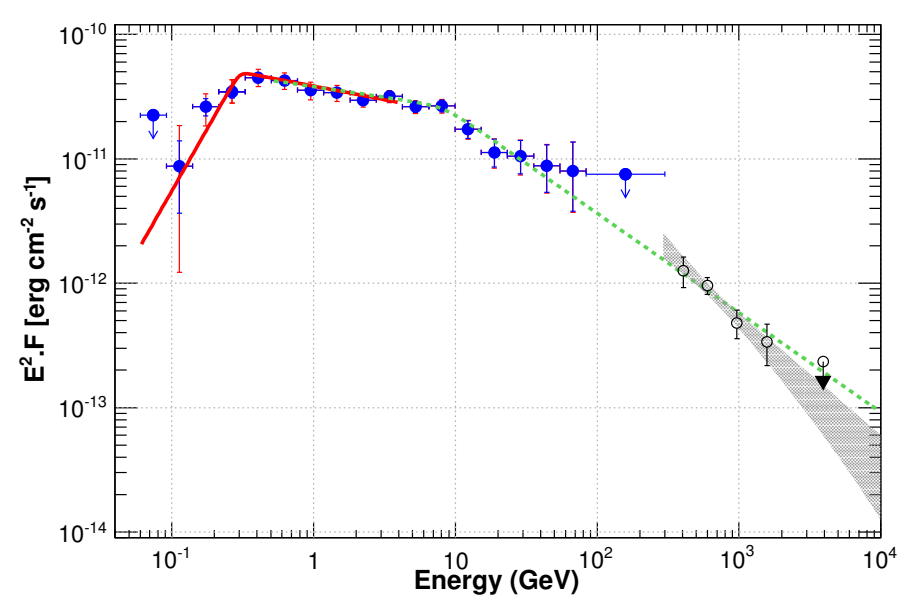

Fig. 3. Fermi-LAT and H.E.S.S. spectrum of W49B. The red line shows the best fit of a smoothly broken power law derived between $60 \mathrm{MeV}$ and $4 \mathrm{GeV}$ and the blue data points indicate the fluxes measured in each energy bin with the Fermi-LAT. The statistical errors are shown in blue, while the red lines take both the statistical and systematic errors into account as discussed in Sect. 2.2.2. The grey band shows the $68 \%$ confidence level (CL) uncertainty of the best-fit power-law model with H.E.S.S. The open black circles indicate the spectral points computed from the forward-folding fit with their statistical errors shown in black. For both instruments, a 95\% CL upper limit is computed when the statistical significance is lower than $2 \sigma$. The dotted green line shows the best smoothly broken power-law model obtained from the joint fit of the Fermi-LAT and H.E.S.S. data between $500 \mathrm{MeV}$ and $10 \mathrm{TeV}$, as described in Sect. 2.3.

than $300 \mathrm{GeV}$, as described by Atwood et al. (2009). It contains a high-resolution converter/tracker (for direction measurement of the incident $\gamma$ rays), a $\mathrm{CsI}(\mathrm{Tl})$ crystal calorimeter (for energy measurement), and an anti-coincidence detector to identify the background of charged particles. The following analysis was performed using five years of Fermi-LAT data collected primarily in survey mode (2008 August 04-2013 August 04). This new data set consists of Pass 7 LAT data that have been reprocessed using updated calibration constants for the detector systems (Bregeon et al. 2013). The primary differences with respect to the Pass7_V6 instrument response functions (IRFs) are the correction of a slight ( $1 \%$ per year) expected degradation in the calorimeter light yield and significant improvement of the position from the calorimeter reconstruction, which in turn significantly improves the LAT point spread function above $5 \mathrm{GeV}$. Only $\gamma$-rays in the Source class events were selected, excluding those coming from a zenith angle larger than $100^{\circ}$ with respect to the detector axis so as to reduce $\gamma$-ray contamination from protons interacting with the atmosphere of the Earth. The 15th version of the reprocessed Pass 7 IRFs for this class of events (P7REP_SOURCE_V15; Bregeon et al. 2013) were adopted.

Two different tools were used to perform the spatial and spectral analysis: gtlike and pointlike. The tool gtlike is a binned maximum likelihood method (Mattox et al. 1996) implemented in the Science Tools distributed by the Fermi Science Support Center (FSSC) ${ }^{2}$. The tool pointlike is an alternate binned likelihood technique, optimised for characterising the extension of a source, a functionality that is not implemented in gtlike. The pointlike tool was extensively tested against gtlike (Lande et al. 2012). These tools fit a source model to the data along with models for isotropic $\gamma$-ray emission that also accounts for the residual charged particles and a diffuse

\footnotetext{
2 More information about the performance of the LAT can be found at the FSSC (http://fermi.gsfc.nasa.gov/ssc).
}

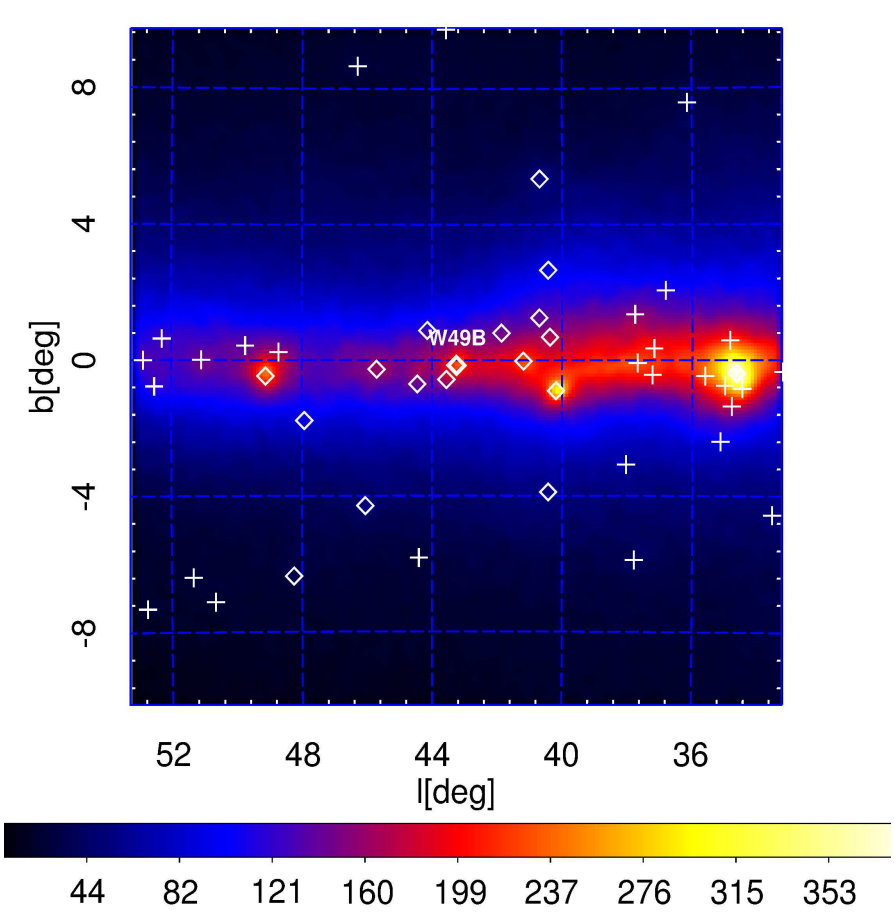

Fig. 4. Fermi-LAT count map of the $20^{\circ} \times 20^{\circ}$ around W49B between $60 \mathrm{MeV}$ and $300 \mathrm{GeV}$, smoothed with a Gaussian of width $0.3^{\circ}$. Spectral parameters of sources indicated by a diamond are left free in the analysis while sources indicated with crosses are frozen in the likelihood fit. Events were spatially binned in pixels of side-length $0.1^{\circ}$. The dominant source seen at a Galactic longitude of $34.6^{\circ}$ in this counts map is W44.

$\gamma$-ray emission model. The obtained log likelihood is then compared between different source model assumptions to find an optimal description of the region. For quantitative evaluation between models, the test-statistic value $(T S)$ was defined as $2 \log \left(\mathcal{L}_{1} / \mathcal{L}_{0}\right)$, where $\mathcal{L}_{1 / 0}$ corresponds to the likelihood value for the source/no-source hypothesis (Mattox et al. 1996). The TS value is a measure of the detection significance or description improvement.

For this work, the Galactic diffuse emission model gll_iem_v05_rev1.fits and isotropic diffuse model iso_source_v05.txt provided by the Fermi-LAT collaboration were used ${ }^{3}$. A $20^{\circ} \times 20^{\circ}$ region of interest binned in $0.1^{\circ}$ bins and centred on the position of W49B was defined. Sources within $25^{\circ}$ of W49B were extracted from the Fermi-LAT 3FGL catalogue (Acero et al. 2015) and included in the likelihood fit. The spectral parameters of sources closer than $5^{\circ}$ to W49B or with a TS larger than 400 were left free and shown with diamonds in Fig. 4, while the parameters of all other sources were fixed at the values from the 3FGL catalogue.

Two main systematic errors have been taken into account: imperfect modelling of the Galactic diffuse emission and uncertainties in the effective area calibration. The first was estimated by comparing the results obtained using the standard Galactic diffuse model with the results based on eight alternative interstellar emission models (IEMs) as performed in de Palma et al. (2013). The alternative IEMs do not necessarily bracket the standard IEM, but provide a good insight into deviations from fit results obtained by the standard IEM expected from variations in the IEM. Comparing results between analyses performed using the standard and alternative IEMs is currently the best method to estimate systematic uncertainties related to

3 These models are available at: http://fermi.gsfc.nasa.gov/ ssc/data/access/lat/BackgroundModels.html 
the IEM modelling. The second major systematic uncertainty was estimated using modified IRFs whose effective areas bracket the nominal areas. These bracketing IRFs are defined by envelopes above and below the nominal energy dependence of the effective area by linearly connecting differences of $(10 \%, 5 \%$, $5 \%$, and $15 \%)$ at $\log _{10}(E / \mathrm{MeV})$ of $(2,2.5,4$, and 6$)$, respectively ${ }^{4}$.

\subsubsection{Fermi-LAT analysis results}

The spatial analysis was performed using all events above $1 \mathrm{GeV}$, thus providing a good compromise between statistics and angular resolution. The source position, determined by pointlike is $\left(\ell=43.251^{\circ}, b=-0.157^{\circ}\right)$ with a statistical uncertainty of $0.015^{\circ}(95 \% \mathrm{CL})$, which is compatible with the radio shell of W49B (see Fig. 2). At this position, the TS value above $1 \mathrm{GeV}$ is 1504 . No significant extension was found with pointlike.

The spectral analysis was then performed with gtlike including all events between 0.06 and $300 \mathrm{GeV}$. Here the bestfit point-source position for $\mathrm{W} 49 \mathrm{~B}$ obtained by pointlike was used in the log likelihood fit. Figure 3 shows the spectral energy distribution obtained for W49B through the maximum likelihood estimation. To derive the flux points, the $0.06-300 \mathrm{GeV}$ range was divided into 20 logarithmically spaced energy bins and a maximum likelihood spectral analysis was performed in each interval, assuming a power-law shape with a fixed spectral index of $\Gamma=2$ for W49B. The normalisations of the diffuse Galactic and isotropic emission were left free in each energy bin as well as those of sources located within $5^{\circ}$ from W49B. A 95\% CL upper limit is computed when the statistical significance is lower than $2 \sigma$. At the highest energies, bins containing no significant signal were combined to compute a meaningful upper limit. The errors on the spectral points represent the statistical and systematic uncertainties added in quadrature.

The spectrum below $300 \mathrm{MeV}$ is steeply rising, clearly exhibiting a break at around $300 \mathrm{MeV}$. To quantify the significance of this spectral break, a log likelihood fit of W49B between $60 \mathrm{MeV}$ and $4 \mathrm{GeV}$ was performed (below the high-energy break previously found in the one-year spectra, Abdo et al. 2010d) using both a single power law of the form $F(E)=$ $\Phi_{0}\left(E / E_{0}\right)^{-\Gamma_{1}}$ and a smoothly broken power law of the form $F(E)=\Phi_{0}\left(E / E_{0}\right)^{-\Gamma_{1}}\left(1+\left(E / E_{\mathrm{br}}\right)^{\left(\Gamma_{2}-\Gamma_{1}\right) / \alpha}\right)^{-\alpha}$, where $\Phi_{0}$ is the differential flux at $E_{0}=200 \mathrm{MeV}$ and $\alpha=0.1$, as was carried out previously for the cases of IC 443 and W44 (Ackermann et al. 2013). The improvement in log likelihood when comparing the broken power law to a single power law corresponds to a formal statistical significance of $8 \sigma$ for the low-energy break (TS of 84 for 2 additional degrees of freedom), as reported in Table 1. Another fit in the same energy band using a log parabola was also performed, showing that the log parabola improves the likelihood by only $5 \sigma$ (TS of 28 for 1 additional degree of freedom). Therefore the best spectral shape for this energy band is the broken power law.

\subsection{H.E.S.S. and Fermi-LAT combined results}

The best-fit position obtained with the Fermi-LAT is coincident with the radio shell, while the fitted position in the $\mathrm{TeV}$ range is more compatible with its centre (see Fig. 2). However, the size of the shell detected at radio wavelengths is comparable to the size of the H.E.S.S. point spread function, preventing discrimination

\footnotetext{
4 http://fermi.gsfc.nasa.gov/ssc/data/analysis/LAT_ caveats.html
}

between the emission originating from the centre of the remnant or from its shell. Furthermore, the spectra derived in the $\mathrm{GeV}$ and $\mathrm{TeV}$ energy ranges are in good agreement (see Fig. 3). The positions and spectra are also compatible with those reported in the recently published second Fermi-LAT catalogue of sources above $50 \mathrm{GeV}$ (Ackermann et al. 2016). It is therefore reasonable to assume that the detected $\gamma$-ray photons are emitted in the same physical region of the W49B remnant.

Taking advantage of the smooth connection between the HE and VHE spectra, the presence of another break at high energy was evaluated through a likelihood ratio test statistic applied to both the H.E.S.S. and Fermi-LAT data. For this test, a single power law and a smoothly broken power law between $500 \mathrm{MeV}$ and $10 \mathrm{TeV}$, which is above the low energy break found above, were used, and the statistical and systematic uncertainties were taken into account. The minimum of the likelihood ratio is reached at $8.4 \mathrm{GeV}$ with a $6.5 \sigma$ improvement of the smoothly broken power-law model with respect to the power-law model. The $68 \%$ confidence interval is $[5.9 ; 10.6] \mathrm{GeV}$ and the spectral indices below and above the break are $2.17 \pm 0.06$ and $2.80 \pm 0.04$, respectively. This result was checked by fitting the Fermi-LAT fluxes of W49B between $500 \mathrm{MeV}$ and $300 \mathrm{GeV}$ with both a single power law and a smoothly broken power law. A $3.3 \sigma$ (TS of 14 for 2 additional degrees of freedom) improvement is obtained for an energy break at $9.4 \pm 2.9_{\text {stat }} \pm 3.8_{\text {sys }} \mathrm{GeV}$. This energy, as well as the spectral indices above and below the break (listed in Table 1), are compatible with the values obtained from the Fermi-LAT and H.E.S.S. joint fit.

\section{Discussion of the results}

\subsection{General considerations}

A spectral break is detected at $304 \pm 20 \mathrm{MeV}$ with the FermiLAT, with properties (break energy, spectral indices) similar to those detected in the SNRs IC 443 and W44 (Ackermann et al. 2013) and interpreted as the signature of $\gamma$-ray emission produced through neutral-pion decay. In addition, the H.E.S.S. and Fermi-LAT joint spectral fit allowed for the estimation of a second spectral break at $8.4_{-2.5}^{+2.2} \mathrm{GeV}$, which is compatible with the break energy position found using the Fermi-LAT data alone $(9.4 \pm 2.9 \mathrm{GeV})$. Such a spectral break has been found in several other interacting SNRs, for instance in W51C (Abdo et al. 2009b), W28 (Abdo et al. 2010a), and G349.7+0.2 (H.E.S.S. Collaboration 2015). The energy at which this break occurs is found to be in the range $1-20 \mathrm{GeV}$, except for $\mathrm{G} 349.7+0.2$ where it is found at a higher energy $(\sim 50 \mathrm{GeV})$. Interestingly, while W49B is estimated to be 1-4 thousand years old, it exhibits spectral features similar to the other detected $\mathrm{SNR} / \mathrm{MC}$ systems that are believed to be $\sim 10$ times older. Likewise, G349.7+0.2 which has roughly the same age as W49B, shows an energy break that is $\sim 5$ times higher. This emphasises the importance of the environment in the evolution of the $\gamma$-ray luminosity of these objects.

The origin of the break in the $1-20 \mathrm{GeV}$ range has been the subject of several theoretical works. It could be explained either by the diffusion of particles escaping the shock (e.g. Ohira et al. 2011; Li \& Chen 2012), acceleration and wave evanescence effects (e.g. Inoue et al. 2010; Malkov et al. 2012), or time-limited re-acceleration of CRs already present in the surroundings (e.g. Uchiyama et al. 2010; Cardillo et al. 2016). Other phenomena might be at play in such objects, such as a so-called neutral return flux that can modify the dynamics of the shock when propagating into a partially neutral medium 
Table 1. Spectral parameters in the energy ranges of $60 \mathrm{MeV}-4 \mathrm{GeV}$ and $500 \mathrm{MeV}-300 \mathrm{GeV}$ for power-law (PL) and smoothly broken power-law (SBPL) models, using Fermi-LAT data and $290 \mathrm{GeV}-10 \mathrm{TeV}$ with H.E.S.S.

\begin{tabular}{lccccc}
\hline \hline Model & $\Phi_{0}$ & $\Gamma_{1}$ & $\Gamma_{2}$ & Ebr & Source TS \\
\hline Fermi-LAT: & & & & \\
60 MeV-4 GeV: & $\left(10^{-10} \mathrm{~cm}^{-2} \mathrm{~s}^{-1} \mathrm{MeV}^{-1}\right)$ & & & $(\mathrm{MeV})$ & \\
PL & $6.2 \pm 0.3$ & $2.07 \pm 0.04$ & $\ldots$ & $\ldots$ & 906 \\
SBPL & $3.3 \pm 0.5( \pm 0.6)$ & $0.10 \pm 0.30( \pm 0.28)$ & $2.21 \pm 0.05( \pm 0.06)$ & $304 \pm 20( \pm 10)$ & 990 \\
\cline { 2 - 4 } $500 \mathrm{MeV}-300 \mathrm{GeV}:$ & $\left(10^{-10} \mathrm{~cm}^{-2} \mathrm{~s}^{-1} \mathrm{MeV}^{-1}\right)$ & & & \\
PL & $10.4 \pm 0.8$ & $2.32 \pm 0.03$ & $\ldots$ & $\ldots$ & 1570 \\
SBPL & $8.2 \pm 1.2( \pm 2.3)$ & $2.20 \pm 0.06( \pm 0.16)$ & $2.89 \pm 0.23( \pm 0.15)$ & $9.4 \pm 2.9( \pm 3.8)$ & 1584 \\
\hline H.E.S.S.: & $\left(10^{-13} \mathrm{~cm}^{-2} \mathrm{~s}^{-1} \mathrm{TeV}^{-1}\right)$ & & & \\
>290 GeV: & $3.15 \pm 0.46( \pm 0.63)$ & $3.14 \pm 0.24( \pm 0.10)$ & $\ldots$ & $\ldots$ & \\
PL & & & & & \\
\hline Fermi-LAT \& H.E.S.S.: & $\left(10^{-10} \mathrm{~cm}^{-2} \mathrm{~s}^{-1} \mathrm{MeV}^{-1}\right)$ & & & & \\
500 MeV-10 TeV: & $7.5 \pm 1.2$ & $2.17 \pm 0.06$ & $2.80 \pm 0.04$ & $8.4_{-2.5}^{+2.2}$ & \\
SBPL & & & & & \\
\hline
\end{tabular}

Notes. Systematic errors are provided in parentheses. The reference energy for which the $\Phi_{0}$ is given is $1 \mathrm{TeV}$ for H.E.S.S. and $200 \mathrm{MeV}$ for Fermi-LAT and the combined spectrum results. The last column indicates the source TS as described in Sect. 2.2.

(Blasi et al. 2012). The maximum energy that accelerated particles can achieve could be limited by the ion-neutral damping (Ptuskin \& Zirakashvili 2005) and their spectrum could be steepened due to the feedback of amplified magnetic fields (Ptuskin et al. 2010; Caprioli 2012). Future precision modelling may be able to take the whole history and environment of W49B into account to predict the spectra of accelerated particles in this source and resultant radiation from radio to $\mathrm{TeV}$ energies. In this work, a simple one-zone model that assumes an empirical distribution of accelerated particles is used specifically to constrain the energetics and the dominant processes in the source.

\subsection{Molecular gas density estimates}

In order to use appropriate values for the modelling, the mass and density of the molecular gas inside a spherical volume of radius $0.025^{\circ}$, which is consistent with that of the radio SNR radius, were estimated using the Galactic Ring Survey ${ }^{13} \mathrm{CO}$ data (Jackson et al. 2006). For the three velocity ranges (1-15, $38-47$, and $57-67 \mathrm{~km} \mathrm{~s}^{-1}$ at their kinematic distances of 11 , 9.3, and $5 \mathrm{kpc}$, respectively) examined by Zhu et al. (2014) (see also Chen et al. 2014), and using the ${ }^{13} \mathrm{CO}$ "X-factor" of $4.92 \times$ $10^{20}(\mathrm{~K} \mathrm{~km} / \mathrm{s})^{-1}$ from Simon et al. (2001), masses of $5 \times 10^{3}$ to $17 \times 10^{3} M_{\odot}$ with densities of 400 to $4000 \mathrm{~cm}^{-3}$ were obtained. Allowing for a larger radius to the outer edge of the radio SNR features $\left(0.05^{\circ}\right)$ reduces the molecular gas densities by a factor $\sim 2$ to 3 .

Chen et al. (2014) and Zhu et al. (2014) have argued that the $\mathrm{CO}$ bubble in the $38-47 \mathrm{~km} \mathrm{~s}^{-1}$ range was blown out by the W49B progenitor star, suggesting a distance of about $10 \mathrm{kpc}$ for the SNR. Additionally for this, and the $57-67 \mathrm{~km} \mathrm{~s}^{-1}$ ranges, the $\mathrm{H}_{2}$ column densities (about $5 \times 10^{22} \mathrm{~cm}^{-2}$ ) inferred in this work are consistent with that derived from the X-ray spectral studies of Keohane et al. (2007).

\subsection{High-energy particles in W49B}

The observed $\gamma$-ray emission is most likely to originate from SNR W49B, as discussed in Abdo et al. (2010d). In this section, the properties of the relativistic particles that radiate at $\gamma$ ray energies are investigated. Given the smooth connection of the observed Fermi-LAT and H.E.S.S. spectra (see Fig. 3), it is assumed that the $\gamma$-ray photons are emitted in the same region. In addition, the observed radio emission(e.g. Moffett \& Reynolds 1994) is assumed to come from the same shell region where a constant hydrogen density $n_{\mathrm{H}}$ and magnetic field strength $B$ are considered. Given the evidence that W49B interacts with the surrounding molecular clouds(e.g. Keohane et al. 2007; Zhu et al. 2014), we consider a scenario where the $\gamma$-ray emission comes from the shocked molecular clouds in the SNR shell. The emitting region may originate from swept-up stellar wind and/or interstellar gas as discussed in Abdo et al. (2010d). However, adopting such a scenario would not significantly change the spectra calculated in this section.

The $\gamma$-ray emission is reproduced using the (relatively) simple model described in Abdo et al. (2009b). Empirical injection distributions of protons and electrons are assumed as follows:

$Q_{\mathrm{e}, \mathrm{p}}(p)=a_{\mathrm{e}, \mathrm{p}}\left(\frac{p}{p_{0}}\right)^{-s}\left(1+\left(\frac{p}{p_{\mathrm{br}}}\right)^{2}\right)^{-\Delta s / 2}$,

where $p_{0}=1 \mathrm{GeV} c^{-1}, p_{\text {br }}$ is the break momentum, $s$ is the spectral index below $p_{\text {br }}$, and $\Delta s$ is the difference between the spectral indices values below and above $p_{\text {br }}$. The kinetic equation for the momentum distribution of high-energy particles in the shell can be written as

$\frac{\partial N_{\mathrm{e}, \mathrm{p}}}{\partial t}=\frac{\partial}{\partial p}\left(b_{\mathrm{e}, \mathrm{p}} N_{\mathrm{e}, \mathrm{p}}\right)+Q_{\mathrm{e}, \mathrm{p}}$

where $b_{\mathrm{e}, \mathrm{p}}=-\mathrm{d} p / \mathrm{d} t$ is the momentum loss rate; only the synchrotron, inverse-Compton, bremsstrahlung, and neutral pion decay radiative losses were considered; and $Q_{\mathrm{e}, \mathrm{p}}(p)$, which is assumed to be time independent, is the particle injection rate. To obtain the radiation spectrum from the remnant, $N_{\mathrm{e}, \mathrm{p}}\left(p, T_{0}\right)$ is numerically calculated for $T_{0}=2000 \mathrm{yr}$.

The $\gamma$-ray emission mechanisms include the $\pi^{0}$-decay $\gamma$-rays due to high-energy protons, bremsstrahlung, and inverseCompton scattering processes by high-energy electrons. The 
Table 2. Parameters for the hadronic scenario.

\begin{tabular}{lcccccc}
\hline \hline Model & $\begin{array}{c}n_{\mathrm{H}} \\
\left(\mathrm{cm}^{-3}\right)\end{array}$ & $s$ & $\Delta s$ & $\begin{array}{c}p_{\mathrm{br}} \\
\left(\mathrm{GeV} \mathrm{c}^{-1}\right)\end{array}$ & $\begin{array}{c}B \\
(\mu \mathrm{G})\end{array}$ & $\begin{array}{c}W_{p} \\
\left(10^{49} \mathrm{erg}\right)\end{array}$ \\
\hline (a1) & 100 & 1.8 & 1.0 & 15 & 100 & 12 \\
(a2) & 100 & 2.0 & 0.8 & 30 & 100 & 11 \\
(a3) & 100 & 2.2 & 0.7 & 100 & 90 & 12 \\
(a4) & 1000 & 1.8 & 1.0 & 15 & 500 & 1.1 \\
(a5) & 1000 & 2.0 & 0.8 & 30 & 500 & 1.1 \\
(a6) & 1000 & 2.2 & 0.7 & 100 & 400 & 1.1 \\
\hline
\end{tabular}

Notes. $K_{\text {ep }}$ is set to 0.01 . The total kinetic energies $\left(E_{\text {kin }}\right)$ of the radiating particles $\left(W_{\mathrm{e}, \mathrm{p}}\right)$ are calculated for $E_{\mathrm{kin}}>100 \mathrm{MeV}$ at an assumed distance of $10 \mathrm{kpc}$.

radio emission is calculated by considering the synchrotron radiation from the population of high-energy electrons. To reproduce the multi-wavelength spectra, two cases of the relativistic electrons to protons number ratio $\left(K_{\mathrm{ep}} \equiv a_{\mathrm{e}} / a_{\mathrm{p}}\right)$ were considered, $K_{\text {ep }}=0.01$ and $K_{\text {ep }}=1$. The value of 0.01 is similar to what is locally observed for cosmic rays at $\mathrm{GeV}$ energies (e.g. Beischer et al. 2009) whereas $K_{\text {ep }}=1$ corresponds to an upper limit of the ratio that is most likely too high for realistic applications due to the faster cooling of the electrons compared to the protons. In the calculation, the same indices and break momenta for both the electrons and the protons were chosen. Given that the radio synchrotron index $\alpha=0.48$ (Moffett $\&$ Reynolds 1994) corresponds to $s \sim 2$, three values for $s$ were considered: $1.8,2.0$, and 2.2. The observed $\gamma$-ray spectra are calculated by adjusting spectral parameters of the relativistic particles: the normalisation, the break momentum $p_{\text {br }}$, and $\Delta s$. The magnetic field $B$ is then determined to explain the observed radio data. Only thermal X-rays, which are not useful in constraining the non-thermal electron population energy distribution, are observed from the SNR (Hwang et al. 2000). In the energy range $1-10 \mathrm{keV}$, our model synchrotron $\mathrm{X}$-ray predictions would be at least one order of magnitude below the measured thermal X-ray values; thus no constraining limits exist in this part of the spectrum. Two conservative cases of $100 \mathrm{~cm}^{-3}$ and $1000 \mathrm{~cm}^{-3}$ for the ambient medium density $n_{\mathrm{H}}$ were considered (see Sec. 3.2). The seed photons for inverse-Compton scattering processes include infrared $\left(k T_{\mathrm{IR}}=3 \times 10^{-3} \mathrm{eV}, U_{\mathrm{IR}}=0.7 \mathrm{eV} \mathrm{cm}^{-3}\right)$, optical $\left(k T_{\text {opt }}=0.3 \mathrm{eV}, U_{\text {opt }}=0.8 \mathrm{eV} \mathrm{cm}^{-3}\right)$, and the CMB. These values were taken to match the axisymmetric estimates of the GALPROP code at the assumed distance of W49B (Porter et al. 2008, and references therein). For a more precise modelling, dedicated analysis of the infrared and optical emission around the source should be performed. The derived parameters and spectra are shown in Tables 2 and 3, and Figs. 5 and 6.

In the case of $K_{\text {ep }}=0.01$ (the hadronic scenario), the obtained $\gamma$-ray spectrum is dominated by the $\pi^{0}$-decay emission due to the relativistic protons as shown in Fig. 5. The observed spectra can be formally explained in all the cases except for the case (a4) where the reproduced synchrotron spectrum seems a little harder than the observed radio data. The rather high values derived for the magnetic field of models (a4-6) are needed to explain the radio data. This is because $K_{\text {ep }}$ is fixed to 0.01 ; allowing for higher values of $K_{\text {ep }}$ would imply higher total kinetic energies for the electrons and lower magnetic field values would be needed to explain the radio data.

The reproduced spectral shape is independent of the value of $n_{\mathrm{H}}$, as long as the range is set to reasonable values $\left(n_{\mathrm{H}} \sim\right.$ $\left.100-1000 \mathrm{~cm}^{-3}\right)$.
Table 3. Parameters for the leptonic scenario.

\begin{tabular}{lcccccc}
\hline \hline Model & $\begin{array}{c}n_{\mathrm{H}} \\
\left(\mathrm{cm}^{-3}\right)\end{array}$ & $s$ & $\Delta s$ & $\begin{array}{c}p_{\text {br }} \\
\left(\mathrm{GeV} \mathrm{c} c^{-1}\right)\end{array}$ & $\begin{array}{c}B \\
(\mu \mathrm{G})\end{array}$ & $\begin{array}{c}W_{\mathrm{e}} \\
\left(10^{49} \mathrm{erg}\right)\end{array}$ \\
\hline (b1) & 100 & 1.8 & 1.1 & 7 & 22 & 1.6 \\
(b2) & 100 & 2.0 & 0.9 & 10 & 25 & 1.6 \\
(b3) & 100 & 2.2 & 0.8 & 30 & 22 & 2.0 \\
(b4) & 1000 & 1.8 & 0.9 & 7 & 100 & 0.16 \\
(b5) & 1000 & 2.0 & 0.7 & 10 & 100 & 0.16 \\
(b6) & 1000 & 2.2 & 0.5 & 15 & 90 & 0.19 \\
\hline
\end{tabular}

Notes. Same as Table 2 except for $K_{\text {ep }}$, which is set to 1 .

In the case of $K_{\mathrm{ep}}=1$ (the leptonic scenario), the obtained $\gamma$-rays are dominated by the bremsstrahlung emission due to the relativistic electrons as shown in Fig. 6. The figure shows that $s \lesssim 1.8$ is required to explain the LAT data below $\sim 300 \mathrm{MeV}$. The synchrotron radiation loss modifies the reproduced spectra at the $\gtrsim \mathrm{TeV}$ range; its break momentum of the electrons are $16 \mathrm{TeV} c^{-1}$ and $0.6 \mathrm{TeV} c^{-1}$ for $B$ of $20 \mu \mathrm{G}$ (corresponding to the cases (b1-3)) and $100 \mu \mathrm{G}$ (corresponding to the cases (b4-6)), respectively. On the other hand, the spectral shape does not significantly change in the energy band below the break.

As discussed in Sect. 2.2, the LAT data show a clear spectral break at $304 \pm 20 \mathrm{MeV}$. Given the systematic errors of the data, both the hadronic and leptonic scenarios can explain this spectral shape. In the leptonic scenario, however, a limit to the spectral index of $s \lesssim 1.8$ must be set to reproduce such a sharp break. On the other hand, the hadronic scenario can naturally explain the break by the $\pi^{0}$-decay bump irrespective of the spectral index. The obtained $B$-field values in Tables 2 and 3 are significantly larger than typical interstellar values. To some extent, these values correspond to those expected in a MC (Crutcher et al. 2010). They are also consistent with predictions of magnetic field amplification from cosmic-ray-excited turbulence (Bell 2004; Zirakashvili et al. 2008). The values adopted in this modelling are set to reasonable values and different assumptions for the parameters (age, distance, $n_{\mathrm{H}}$, and $K_{\mathrm{ep}}$ ) would change the quantitative results of the modelling slightly (derived magnetic field or energy values), but would not change these conclusions.

\section{Conclusions}

A source of very high-energy $\gamma$ rays is detected towards the supernova remnant W49B with H.E.S.S. and a joint study is conducted together with the analysis of five years of Fermi-LAT data towards the source. The point spread functions of these instruments are comparable to the physical size of the SNR and do not allow for detailed morphology studies. However, the significantly increased data set compared to previous publications allowed for the derivation of the $\gamma$-ray spectrum of the source between $60 \mathrm{MeV}$ and a few TeV. Two spectral breaks could be identified in the $\gamma$-ray spectrum: one at $304 \mathrm{MeV}$ and the other at $8.4 \mathrm{GeV}$. The latter, constrained from a joint fit of the Fermi-LAT and H.E.S.S. data, is similar to the spectral breaks observed in other supernova remnants interacting with molecular clouds and further supports the evidence of interaction observed in other wavelengths for this object. The broadband spectrum of W49B could be explained either by leptonic or hadronic models. However, the sharp break detected with the Fermi-LAT data at $304 \mathrm{MeV}$ seems to favour a hadronic origin of the $\gamma$-ray emission 


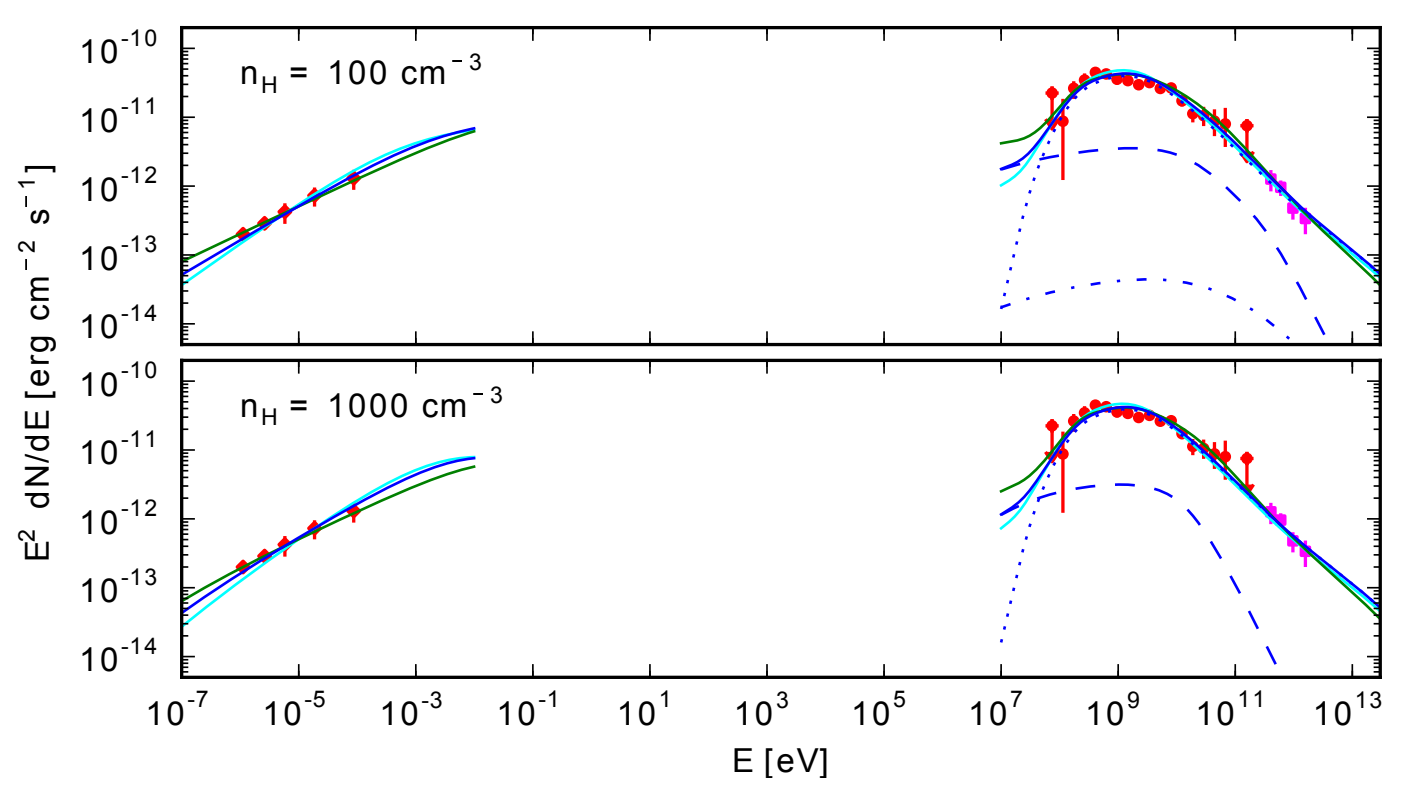

Fig. 5. SEDs of W49B with model curves for the hadronic-dominant scenario. The upper and lower panels show (a1-3) and (a4-6), respectively (see Table 2). The red diamonds, red circles, and magenta squares represent observed data in the radio (Moffett \& Reynolds 1994), LAT, and H.E.S.S. bands, respectively. The radio emission is explained by the synchrotron radiation from the relativistic electrons. The $\gamma$-ray emission can be decomposed into $\pi^{0}$-decay (dotted line), bremsstrahlung (dashed line), and IC scattering (dot-dashed line). The solid line represents the total flux of the components. The cases (a1)/(a4), (a2)/(a5), and (a3)/(a6) are represented by cyan, blue, and green lines, respectively, in the upper/lower panel. The decomposed emissions are shown for the cases (a2) and (a5) in the upper and lower panels, respectively.

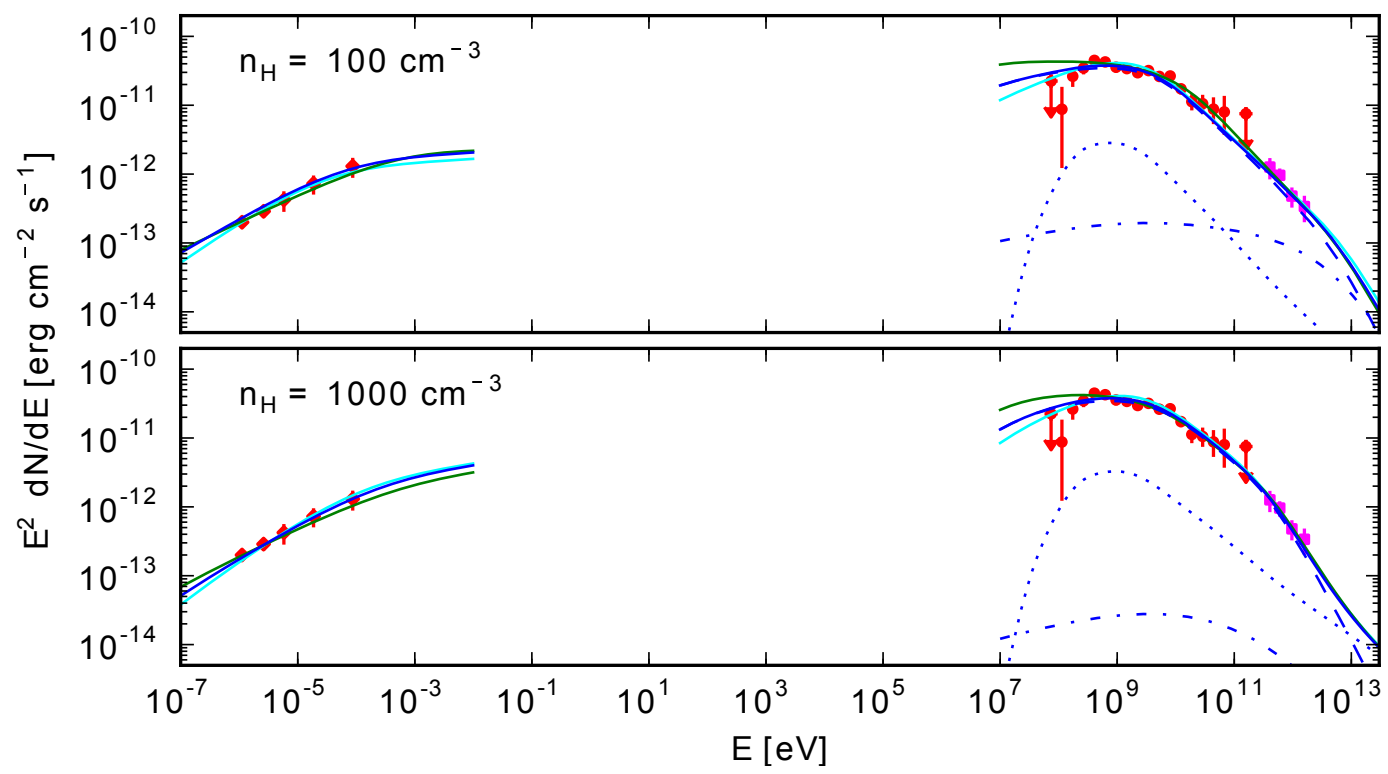

Fig. 6. Same as Fig. 5 but for the leptonic-dominant scenario. The upper and lower panels show reproduced spectra of (b1-3) and (b4-6), respectively (see Table 3). The cases (b1)/(b4), (b2)/(b5), and (b3)/(b6) are represented by cyan, blue, and green lines, respectively, in the upper/lower panel. The decomposed emission is shown for the cases (b2) and (b5) in the upper and lower panels, respectively.

since the leptonic models would require an even harder spectrum for the electron population than those tested in this work, which would fail to explain the radio observations.

In the near future, W49B and the nearby star-forming region W49A will be of particular interest to study at VHE with improved analysis methods and with the next generation of instruments such as the Cherenkov Telescope Array. Observations or analyses of the W49 region with improved angular resolution and higher sensitivity would help to constrain the morphology and the origin of the emission towards W49B and possibly confirm the hint of emission towards W49A. Furthermore, if one assumes that the distances to W49A and W49B are comparable, then the observed difference between the $\gamma$-ray luminosities of the two sources would become especially interesting. This would imply that, in the absence of recognizable supernova remnants, as in W49A, the other possible energetic particle sources, such as shocks expected from interacting or collective stellar winds, do not appear very effective for HE and VHE $\gamma$-ray emission in this case. Therefore, a truly reliable distance determination for these sources (see Sect. 1) would be of great astrophysical importance.

Acknowledgements. The support of the Namibian authorities and of the University of Namibia in facilitating the construction and operation of H.E.S.S. is gratefully acknowledged, as is the support by the German Ministry for Education and Research (BMBF), the Max Planck Society, the German Research 
Foundation (DFG), the French Ministry for Research, the CNRS-IN2P3 and the Astroparticle Interdisciplinary Programme of the CNRS, the UK Science and Technology Facilities Council (STFC), the IPNP of the Charles University, the Czech Science Foundation, the Polish Ministry of Science and Higher Education, the South African Department of Science and Technology and National Research Foundation, the University of Namibia, the Innsbruck University, the Austrian Science Fund (FWF), and the Austrian Federal Ministry for Science, Research and Economy, and by the University of Adelaide and the Australian Research Council. We appreciate the excellent work of the technical support staff in Berlin, Durham, Hamburg, Heidelberg, Palaiseau, Paris, Saclay, and in Namibia in the construction and operation of the equipment. This work benefited from services provided by the H.E.S.S. Virtual Organisation, supported by the national resource providers of the EGI Federation. The Fermi LAT Collaboration acknowledges generous ongoing support from a number of agencies and institutes that have supported both the development and the operation of the LAT as well as scientific data analysis. These include the National Aeronautics and Space Administration and the Department of Energy in the United States, the Commissariat à l'Énergie Atomique and the Centre National de la Recherche Scientifique/Institut National de Physique Nucléaire et de Physique des Particules in France, the Agenzia Spaziale Italiana and the Istituto Nazionale di Fisica Nucleare in Italy, the Ministry of Education, Culture, Sports, Science and Technology (MEXT), High Energy Accelerator Research Organization (KEK), and Japan Aerospace Exploration Agency (JAXA) in Japan, and the K. A. Wallenberg Foundation, the Swedish Research Council, and the Swedish National Space Board in Sweden. Additional support for science analysis during the operations phase is gratefully acknowledged from the Istituto Nazionale di Astrofisica in Italy and the Centre National d'Études Spatiales in France. This publication makes use of molecular line data from the Boston University-FCRAO Galactic Ring Survey (GRS). The GRS is a joint project of Boston University and Five College Radio Astronomy Observatory, funded by the Nationa Science Foundation under grants AST-9800334, AST-0098562, AST-0100793, AST-0228993 \& AST-0507657.

\section{References}

Abdo, A. A., Ackermann, M., Ajello, M., et al. 2009a, ApJS, 183, 46 Abdo, A. A., Ackermann, M., Ajello, M., et al. 2009b, ApJ, 706, L1 Abdo, A. A., Ackermann, M., Ajello, M., et al. 2010a, ApJ, 718, 348 Abdo, A. A., Ackermann, M., Ajello, M., et al. 2010b, Science, 327, 1103 Abdo, A. A., Ackermann, M., Ajello, M., et al. 2010c, ApJ, 712, 459 Abdo, A. A., Ackermann, M., Ajello, M., et al. 2010d, ApJ, 722, 1303 Abramowski, A., Acero, F., Aharonian, F., et al. 2012, A\&A, 537, A114

Acciari, V. A., Aliu, E., Arlen, T., et al. 2009, ApJ, 698, L133

Acero, F., Ackermann, M., Ajello, M., et al. 2015, ApJS, 218, 23

Ackermann, M., Ajello, M., Allafort, A., et al. 2013, Science, 339, 807

Ackermann, M., Ajello, M., Atwood, W. B., et al. 2016, ApJS, 222, 5

Aharonian, F., Akhperjanian, A. G., Bazer-Bachi, A. R., et al. 2006a, A\&A, 457 899

Aharonian, F., Akhperjanian, A. G., Bazer-Bachi, A. R., et al. 2006b, A\&A, 449, 223

Aharonian, F., Akhperjanian, A. G., Barres de Almeida, U., et al. 2008a, A\&A, 490, 685

Aharonian, F., Akhperjanian, A. G., Bazer-Bachi, A. R., et al. 2008b, A\&A, 481, 401

Aharonian, F., Akhperjanian, A. G., de Almeida, U. B., et al. 2009, ApJ, 692, 1500

Albert, J., Aliu, E., Anderhub, H., et al. 2007, ApJ, 664, L87

Atwood, W. B., Abdo, A. A., Ackermann, M., et al. 2009, ApJ, 697, 1071

Beischer, B., van Doetinchem, P., Gast, H., et al. 2009, New J. Phys., 11, 105021

Bell, A. R. 2004, MNRAS, 353, 550

Berge, D., Funk, S., \& Hinton, J. 2007, A\&A, 466, 1219

Blasi, P. 2013, A\&ARv, 21, 70

Blasi, P., Morlino, G., Bandiera, R., Amato, E., \& Caprioli, D. 2012, ApJ, 755, 121

Bregeon, J., Charles, E., \& M. Wood (for the Fermi-LAT Collaboration) 2013, 2012 Fermi Symp. Proc. eConf. C121028 [arXiv: 1304 . 5456]

Brogan, C. L., \& Troland, T. H. 2001, ApJ, 550, 799

Caprioli, D. 2012, J. Cosmol. Astropart. Phys., 7, 038

Cardillo, M., Amato, E., \& Blasi, P. 2016, A\&A, 595, A58

Castro, D., \& Slane, P. 2010, ApJ, 717, 372

Chen, Y., Jiang, B., Zhou, P., et al. 2014, in IAU Symp. 296, eds. A. Ray, \& R. A. McCray, 170

Crutcher, R. M., Wandelt, B., Heiles, C., Falgarone, E., \& Troland, T. H. 2010, ApJ, 725, 466

de Naurois, M., \& Rolland, L. 2009, Astropart. Phys., 32, 231

de Palma, F., Brandt, T. J., Johannesson, G., Tibaldo, L. (for the FermiLAT Collaboration) 2013, 2012 Fermi Symp. Proc. eConf. C121028 [arXiv: 1304.1395]
Drury, L. O. 1983, Rep. Prog. Phys., 46, 973

González-Casanova, D. F., De Colle, F., Ramirez-Ruiz, E., \& Lopez, L. A. 2014 ApJ, 781, L26

Green, D. A. 2014, Bull. Astron. Soc. India, 42, 47

Helfand, D. J., Becker, R. H., White, R. L., Fallon, A., \& Tuttle, S. 2006, AJ, 131,2525

H.E.S.S. Collaboration (Abramowski, A., Aharonian, F., et al.) 2015, A\&A, 574, A100

H.E.S.S. Collaboration (Abdalla, H., Abramowski, A., et al.) 2018, A\&A, 612, A1 (H.E.S.S. SI)

Hinton, J. A. 2004, New Astron., 48, 331

Hwang, U., Petre, R., \& Hughes, J. P. 2000, ApJ, 532, 970

Inoue, T., Yamazaki, R., \& Inutsuka, S.-I. 2010, ApJ, 723, L108

Jackson, J. M., Rathborne, J. M., Shah, R. Y., et al. 2006, ApJS, 163, 145

Jogler, T., \& Funk, S. 2016, ApJ, 816, 100

Keohane, J. W., Reach, W. T., Rho, J., \& Jarrett, T. H. 2007, ApJ, 654, 938

Lande, J., Ackermann, M., Allafort, A., et al. 2012, ApJ, 756, 5

Li, H., \& Chen, Y. 2012, MNRAS, 421, 935

Li, T.-P., \& Ma, Y.-Q. 1983, ApJ, 272, 317

Lockman, F. J. 1989, ApJS, 71, 469

Lopez, L. A., Ramirez-Ruiz, E., Castro, D., \& Pearson, S. 2013, ApJ, 764, 50

Malkov, M. A., Diamond, P. H., \& Sagdeev, R. Z. 2012, Phys. Plasmas, 19, 082901

Mattox, J. R., Bertsch, D. L., Chiang, J., et al. 1996, ApJ, 461, 396

Mezger, P. G., Schraml, J., \& Terzian, Y. 1967, ApJ, 150, 807

Moffett, D. A., \& Reynolds, S. P. 1994, ApJ, 437, 705

Ohira, Y., Murase, K., \& Yamazaki, R. 2011, MNRAS, 410, 1577

Ohm, S., van Eldik, C., \& Egberts, K. 2009, Astropart. Phys., 31, 383

Parsons, R. D., \& Hinton, J. A. 2014, Astropart. Phys., 56, 26

Piron, F., Djannati-Atai, A., Punch, M., et al. 2001, A\&A, 374, 895

Porter, T. A., Moskalenko, I. V., Strong, A. W., Orlando, E., \& Bouchet, L. 2008 , ApJ, 682, 400

Ptuskin, V. S., \& Zirakashvili, V. N. 2005, A\&A, 429, 755

Ptuskin, V., Zirakashvili, V., \& Seo, E.-S. 2010, ApJ, 718, 31

Pye, J. P., Becker, R. H., Seward, F. D., \& Thomas, N. 1984, MNRAS, 207, 649

Radhakrishnan, V., Goss, W. M., Murray, J. D., \& Brooks, J. W. 1972, ApJS, 24, 49

Rho, J., \& Petre, R. 1998, ApJ, 503, L167

Scoville, N. Z., Sargent, A. I., Sanders, D. B., et al. 1986, ApJ, 303, 416

Simon, R., Jackson, J. M., Clemens, D. P., Bania, T. M., \& Heyer, M. H. 2001, ApJ, 551, 747

Uchiyama, Y., Blandford, R. D., Funk, S., Tajima, H., \& Tanaka, T. 2010, ApJ, 723, L122

Walker, R. C., Matsakis, D. N., \& Garcia-Barreto, J. A. 1982, ApJ, 255, 128

Westerhout, G. 1958, Bull. Astron. Inst. Netherlands, 14, 215

Wu, S.-W., Bik, A., Henning, T., et al. 2014, A\&A, 568, L13

Zhang, B., Reid, M. J., Menten, K. M., et al. 2013, ApJ, 775, 79

Zhou, X., Miceli, M., Bocchino, F., Orlando, S., \& Chen, Y. 2011, MNRAS, 415, 244

Zhu, H., Tian, W. W., \& Zuo, P. 2014, ApJ, 793, 95

Zirakashvili, V. N., Ptuskin, V. S., \& Völk, H. J. 2008, ApJ, 678, 255

1 Centre for Space Research, North-West University, 2520 Potchefstroom, South Africa

2 Universität Hamburg, Institut für Experimentalphysik, Luruper Chaussee 149, 22761 Hamburg, Germany

3 Max-Planck-Institut für Kernphysik, PO Box 103980, 69029 Heidelberg, Germany

4 Dublin Institute for Advanced Studies, 31 Fitzwilliam Place, Dublin 2, Ireland

5 National Academy of Sciences of the Republic of Armenia, Marshall Baghramian Avenue, 24, 0019 Yerevan, Republic of Armenia

6 Yerevan Physics Institute, 2 Alikhanian Brothers St., 375036 Yerevan, Armenia

7 Institut für Physik, Humboldt-Universität zu Berlin, Newtonstr. 15, 12489 Berlin, Germany

8 University of Namibia, Department of Physics, Private Bag 13301, Windhoek, Namibia

9 GRAPPA, Anton Pannekoek Institute for Astronomy, University of Amsterdam, Science Park 904, 1098 XH Amsterdam, The Netherlands

10 Department of Physics and Electrical Engineering, Linnaeus University, 35195 Växjö, Sweden 
11 Institut für Theoretische Physik, Lehrstuhl IV: Weltraum und Astrophysik, Ruhr-Universität Bochum, 44780 Bochum, Germany

12 GRAPPA, Anton Pannekoek Institute for Astronomy and Institute of High-Energy Physics, University of Amsterdam, Science Park 904, 1098 XH Amsterdam, The Netherlands

13 Institut für Astro- und Teilchenphysik, Leopold-FranzensUniversität Innsbruck, 6020 Innsbruck, Austria

14 School of Physical Sciences, University of Adelaide, 5005 Adelaide, Australia

15 LUTH, Observatoire de Paris, PSL Research University, CNRS, Université Paris Diderot, 5 Place Jules Janssen, 92190 Meudon, France

16 Sorbonne Universités, UPMC Université Paris 06, Université Paris Diderot, Sorbonne Paris Cité, CNRS, Laboratoire de Physique Nucléaire et de Hautes Energies (LPNHE), 4 place Jussieu, 75252 Paris Cedex 5, France

${ }^{17}$ Laboratoire Univers et Particules de Montpellier, Université Montpellier, CNRS/IN2P3, CC 72, Place Eugène Bataillon, 34095 Montpellier Cedex 5, France

18 DSM/Irfu, CEA Saclay, 91191 Gif-Sur-Yvette Cedex, France

19 Astronomical Observatory, The University of Warsaw, Al. Ujazdowskie 4, 00-478 Warsaw, Poland

20 Aix Marseille Université, CNRS/IN2P3, CPPM UMR 7346, 13288 Marseille, France

21 Instytut Fizyki Jạdrowej PAN, ul. Radzikowskiego 152, 31-342 Kraków, Poland

22 Funded by EU FP7 Marie Curie, grant agreement No. PIEF-GA-2012-332350

23 School of Physics, University of the Witwatersrand, 1 Jan Smuts Avenue, Braamfontein, 2050 Johannesburg, South Africa

${ }^{24}$ Laboratoire d'Annecy-le-Vieux de Physique des Particules, Université Savoie Mont-Blanc, CNRS/IN2P3, 74941 Annecy-leVieux, France

25 Landessternwarte, Universität Heidelberg, Königstuhl, 69117 Heidelberg, Germany

26 Université Bordeaux, CNRS/IN2P3, Centre d'Études Nucléaires de Bordeaux Gradignan, 33175 Gradignan, France

27 Oskar Klein Centre, Department of Physics, Stockholm University, Albanova University Center, 10691 Stockholm, Sweden

28 Wallenberg Academy Fellow

29 Institut für Astronomie und Astrophysik, Universität Tübingen, Sand 1, 72076 Tübingen, Germany
${ }^{30}$ Laboratoire Leprince-Ringuet, Ecole Polytechnique, CNRS/IN2P3, 91128 Palaiseau, France

31 APC, AstroParticule et Cosmologie, Université Paris Diderot, CNRS/IN2P3, CEA/Irfu, Observatoire de Paris, Sorbonne Paris Cité, 10 rue Alice Domon et Léonie Duquet, 75205 Paris Cedex 13, France

32 Univ. Grenoble Alpes, IPAG CNRS, IPAG, 38000 Grenoble, France

33 Department of Physics and Astronomy, The University of Leicester, University Road, Leicester, LE1 7RH, UK

34 Nicolaus Copernicus Astronomical Center, ul. Bartycka 18, 00-716 Warsaw, Poland

35 Institut für Physik und Astronomie, Universität Potsdam, Karl-Liebknecht-Strasse 24/25, 14476 Potsdam, Germany

${ }^{36}$ Friedrich-Alexander-Universität Erlangen-Nürnberg, Erlangen Centre for Astroparticle Physics, Erwin-Rommel-Str. 1, 91058 Erlangen, Germany

37 DESY, 15738 Zeuthen, Germany

38 Obserwatorium Astronomiczne, Uniwersytet Jagielloński, ul. Orla 171, 30-244 Kraków, Poland

39 Centre for Astronomy, Faculty of Physics, Astronomy and Informatics, Nicolaus Copernicus University, Grudziadzka 5, 87-100 Torun, Poland

40 Department of Physics, University of the Free State, PO Box 339, 9300 Bloemfontein, South Africa

41 Heisenberg Fellow (DFG), ITA Universität Heidelberg, 69029 Heidelberg, Germany

42 GRAPPA, Institute of High-Energy Physics, University of Amsterdam, Science Park 904, 1098 XH Amsterdam, The Netherlands

43 W. W. Hansen Experimental Physics Laboratory, Kavli Institute for Particle Astrophysics and Cosmology, Department of Physics and SLAC National Accelerator Laboratory, Stanford University, Stanford, CA 94305, USA

44 Department of Physics, Rikkyo University, 3-34-1 Nishi-Ikebukuro, Toshima-ku, 171-8501 Tokyo, Japan

45 Department of Physical Sciences, Hiroshima University, Higashi-Hiroshima, 739-8526 Hiroshima, Japan

46 Now at Santa Cruz Institute for Particle Physics and Department of Physics, University of California at Santa Cruz, Santa Cruz, CA 95064, USA 